\title{
Evaluation of the presence of microorganisms in solid-organ preservation solution
}

\section{Authors}

André Marcelo Colvara Mattana ${ }^{1}$

Alexandre Rodrigues Marra² Antônia Maria de Oliveira Machado $^{3}$

Gaspar de Jesus Lopes Filho ${ }^{4}$ Alcides Augusto Salzedas Netto ${ }^{5}$

Adriano Miziara Gonzalez 6

${ }^{1}$ Postgraduate Student, Surgical Gastroenterology Service,

Universidade Federal de São

Paulo - Escola Paulista de

Medicina (UNIFESP-EPM);

General and Digestive Surgeon,

Hospital Santo Antônio and

Hospital Santa Catarina,

Blumenal, SC, Brazil

${ }^{2} \mathrm{PhD}$; Infectologist and

Intensivist, Hospital Israelita

Albert Einstein, SP, Brazil

${ }^{3} \mathrm{PhD}$, Technical Director,

Instituto Paulista de Doenças

Infecciosas e Parasitárias;

Technical Director, Laboratório

Central do Hospital São Paulo,

SP, Brazil

$\mathrm{PhD}$; Associate Professor

UNIFESP; Chief of Surgical

Gastroenterology, Universidade

Estadual Paulista (UNESP), SP,

Brazil

${ }^{5} \mathrm{PhD}$; Surgeon, Kidney

Transplantation Program,

Hospital do Câncer and Hospital

Sírio-Libanês; Assistant of

Pediatric Surgery,

UNIFESP-EPM, SP, Brazil

${ }^{6} \mathrm{PhD}$; Adjunct Professor,

Digestive Transplantation

Coordinator, UNIFESP, SP, Brazi

Submitted on: 03/15/2011

Approved on: 09/09/2011

Correspondence to:

Adriano Miziara Gonzalez

Rua Napoleão de Barros, 610

Vila Clementino

04024-002, São Paulo, SP

Brazil

Phone: +55 11 5084-7551/

5084-9131

amgonzalez@uol.com.br

We declare no conflict of interest.

\begin{abstract}
Objective: To assess the presence of microorganism contamination in the preservation solution for transplant organs (kidney/pancreas). Method: Between August 2007 and March 2008, 136 samples of preservation solution were studied prior to graft implantation. Variables related to the donor and to the presence of microorganisms in the preservation solution of organs were evaluated, after which the contamination was evaluated in relation to the "recipient culture" variable. Univariate and multivariate statistical analyses were performed. Results: The contamination rate of the preservation solution was $27.9 \%$. Coagulase-negative Staphylococcus was the most frequently isolated microorganism. However, highly virulent agents, such as fungi and enterobacteria, were also isolated. In univariate analysis, the variable "donor antibiotic use" was significantly associated to the contamination of the preservation solution. On the other hand, multivariate analysis found statistical significance in "donor antibiotic use" and "donor's infectious complications" variables. Conclusions: In this study, $27.9 \%$ of the preservation solutions of transplant organs were contaminated. Infectious diseases and non-use of antibiotics by the donor were significantly related to the presence of microorganisms in organ preservation solutions. Contamination in organ preservation solutions was not associated with infection in the recipient.
\end{abstract}

Keywords: organ transplantation; disease transmission, infectious; organ preservation solutions; microbial viability.

\section{INTRODUCTION}

Every year, there is an increasing number of transplanted organs, and thus a need for greater organ availability. In the United States, the mortality rate for patients on the waiting list for an organ is around $7 \% .^{1}$ The disparity between organ supply and demand is translated by the use of high-risk donors for patients with advanced disease.

It has been reported that $1.2-62 \%$ of infectious agents, such as Gram-positive and Gram-negative bacteria, as well as fungi, are present in the preservation solution immediately after the collection process, suggesting that this stage is an important source of contamination. ${ }^{2,3}$

The origin of contaminating microorganisms is not always clear. There may be airborne transmission in the surgical environment from the surgical staff, surgical instruments or poor skin antisepsis. ${ }^{4}$ Concerning brain-dead donors, there is a higher risk of infection due to severe impairment of the cellular immune system, as well as hemodynamic instability, and subsequent bacterial translocation from the gut. ${ }^{5}$

\section{METHODS}

This study aims to determine the presence of microbial infection in the preservation solution, prior to solid organ implantation (pancreas and kidney), by analyzing possible factors related to the risk of infection since these data were not found in Brazilian literature.

This study was conducted in the Kidney and Hypertension Hospital (São Paulo), during kidney and pancreas transplants from donors deceased in the period from August 2007 to March 2008

One hundred thirty-six samples of organ preservation solution were prospectively analyzed for the presence of microorganisms prior to transplantation. Each sample of the preservation solution came from an organ (kidney 
or pancreas) collected from a total of 89 deceased donors. This study did not have an interventionist role. All organs were collected in accredited centers in São Paulo, which follow strict rules for excision, storage and transportation. Organs were preserved by using the static hypothermia technique with the organs still inside the donors' bodies (in situ perfusion). After collection of the organs, they were placed in a sterile container (endo-bag), and immersed in preservation solution. They were kept at around $4^{\circ} \mathrm{C}$. For each collected organ, one of the following preservation solutions was used: Soltran ${ }^{\circledR}$, Euro-Collins, Celsior ${ }^{\circledR}$ and Belzer.

Samples of preservation solutions were collected from the container in which the organ was stored at the time of its removal from storage immediately prior to implantation. After homogenization of the solution, $10 \mathrm{~mL}$ was collected with a syringe and placed in a sterile universal collection bottle. No quantitative analysis was performed with these samples, and the liquid was inoculated directly into a suitable medium, which was immediately transported to the hospital laboratory within a maximum interval of 2 hours between sample collection and inoculation into culture media.

Sheep Blood Agar, Sabouraud Agar and Brain-HeartInfusion (BHI) broth were used for sample processing.

After inoculation in the aforementioned culture media, the samples were incubated as described below:

- Blood Agar Medium and BHI broth at $35 \pm 1^{\circ} \mathrm{C}$ for 72 hours, with daily readings.

- Sabouraud Agar medium at room temperature for 30 days, with periodic readings.

- Gram staining was performed on any growth obtained in any culture media, and the isolated contaminating agent was identified through biochemical tests specific for each microorganism according to routine laboratory protocol. Neither antimicrobial susceptibility test (AST) nor microorganism genotyping were performed.

In order to assess the risk factors for the presence of microorganisms in the preservation solution, several variables related to the donor and the organ were evaluated. Among the variables related to the donor, age, duration of ICU stay, duration of antibiotic therapy, cause of death, gender, presence of infection and antibiotic use were considered. The variables associated with the transplanted organs were cold ischemia time, type of organ, and type of preservation solution. The presence of contamination in the preservation solution was then evaluated with the $\mathrm{cul}$ ture of the recipient variable.

All independent variables concerning donors and organs were compared to the dependent variable culture result of the preservation solution, by means of univariate analysis. The independent variable culture of the recipient was also compared to the culture result of the organ preservation solution. Chi-square test and the Student's $t$ test were used for the evaluation of the categorical and quantitative variables, respectively. A p-value lower than 0.05 was considered statistically significant. A logistic regression model was fitted such that all independent variables were compared simultaneously with the dependent variable preservation solution culture. Statistical analyses were performed using the SPSS program version 10.0 (SPSS Statistical Software, Inc, 1999). ${ }^{6}$

The 136 samples of preservation solution for collected organs (kidney or pancreas) from 89 deceased donors were cultured. Of these, 50 donated a single kidney, 26 donated two kidneys, five donated one kidney and a pancreas, and 8 donated two kidneys and a pancreas. In total, 123 recipients benefited from these transplants, with 13 simultaneously receiving both a kidney and a pancreas.

Out of the 136 cultures of preservation solution, 38 (27.9\%) showed growth of some microorganism. The isolated microorganisms are listed in Table 1.

In two recipients with active infectious processes and positive cultures, the same agent previously isolated in the culture of organ preservation solution was observed. In the first case, a urinary tract infection in the recipient caused by Enterococcus sp. was identified. This organism was isolated both in the urine culture of the kidney/pancreas recipient and in the culture of preservation solution. In the second case, an infection of the surgical site caused by Klebsiella pneumoniae was identified. This organism was isolated both in the culture of the surgical wound secretion of the recipient and in the culture of the preservation solution.

In the univariate analysis, the dependent variable culture of preservation solution was assessed against the other independent variables. For statistical purposes, the total number of donors was considered to be 136, i.e., equal to the number of preservation solution samples (136 organs). Importantly, only three cultures from the same donor exhibited the same contaminant bacteria isolated from the organ preservation solutions. In this analysis only the categorical variable use of antibiotics by donor had a statistic significance $(\mathrm{p}=0.0275)$.

By analyzing all evaluated variables (risk factors) simultaneously, a significant of logistic regression model with the variables culture of preservation solution, use of antibiotics by donor (OR - 0.1956, CI (95\%) - 0,0689, p - 0,0022) and infectious occurrences in donor (OR - 3.0495, CI (95\%) $1.1725, \mathrm{p}-0,0222$ ) was obtained. The other variables studied did not add significance to the model.

Using this logistic regression model, a probability matrix was obtained with all possible combinations of the predictor variable categories (infectious occurrences in donor and use of antibiotics by donor), where both predictor variables are dichotomous (yes/ no). In this matrix, there is a $70.1 \%$ chance of a positive preservation solution culture in those cases where the donor had an infection and belonged to the group "not using antibiotics".

Infection is still one of the main causes of morbidity and mortality after organ transplants, even with the use of modern antimicrobial agents. ${ }^{7}$ In solid organ transplants, 
Table 1. Microorganisms isolated in the culture of 136 samples of solid organ preservation solution, prior to implantation of the graft

\begin{tabular}{|c|c|c|c|}
\hline Culture of preservation solution & Microorganisms & Number of organs & $\%$ \\
\hline \multirow[t]{11}{*}{ Without growth } & & 98 & 72.46 \\
\hline & Gram-positive & & \\
\hline & Coagulase-negative Staphylococcus & 11 & 7.97 \\
\hline & Bacillus spp. & 5 & 3.62 \\
\hline & Enterococcus spp. & 5 & 3.62 \\
\hline & Staphylococcus aureus & 2 & 1.45 \\
\hline & Beta-hemolytic Streptococcus & 1 & 0.72 \\
\hline & Gram-negative (monomicrobial) & & \\
\hline & Pseudomonas aeruginosa & 2 & 1.45 \\
\hline & Acinetobacter spp. & 2 & 1.45 \\
\hline & Acinetobacter baumannii & 1 & 0.72 \\
\hline \multicolumn{4}{|l|}{ With growth } \\
\hline & Acinetobacter iwoffii & 1 & 0.72 \\
\hline & Escherichia coli & 1 & 0.72 \\
\hline & Enterobacter spp. & 1 & 0.72 \\
\hline & Klebsiella pneumoniae & 1 & 0.72 \\
\hline \multicolumn{4}{|c|}{ Fungi } \\
\hline & Candida krusei & 2 & 1.45 \\
\hline & Candida parapsilosis & 1 & 0.72 \\
\hline & $\begin{array}{l}\text { Candida parapsilosis / Escherichia coli } \\
\text { (polymicrobial) }\end{array}$ & 2 & 1.45 \\
\hline Total & & 136 & 100.00 \\
\hline
\end{tabular}

infectious processes are responsible for the majority of graft losses despite new immunosuppression protocols and efforts to identify infectious agents in both the donor and the recipient. ${ }^{8-10}$ Prospective studies have reported mortality rates after transplants around $50 \%$ in the case of invasive fungal processes and up to $30 \%$ in the case of bacterial infections. ${ }^{11}$ Up to $75 \%$ of transplanted patients will present some infectious process in the first year after transplant. ${ }^{12}$

In Brazil, Linhares et al. ${ }^{13}$ demonstrated that in a series of 45 combined pancreas/kidney transplants, $51 \%$ of infectious events required hospitalization, $71 \%$ of which were bacterial, $16 \%$ viral and 13\% fungal. Urinary and surgical wound infections were the most frequent infection foci. Infectious complications accounted for $50 \%$ mortality.

Today, there is a growing imbalance between supply and demand of organs. The use of non-ideal donors, such as those with documented infection, is becoming necessary. ${ }^{10,14}$ Thus, the use of organs collected from donors with active bacterial and/or fungal infections, provided they are controllable, does not correlate to a significant increase in the number of complications in the recipient when routine antibiotic prophylaxis is administered., ${ }^{415}$ There are few re- ports in the literature of the transmission of non-viral infectious agents through the preservation solution during solid organ transplant with deceased donors. However, when present, these can be catastrophic. It is very difficult to differentiate the possible sources for the emergence of the infectious agent since its origin may be from the donor, exogenous (contamination), and/or reactivation of a latent infection in the recipient. ${ }^{16}$

Preservation solutions may keep microorganisms viable, facilitate their growth, and even mediate an invasive infectious process in an immunocompromised recipient. ${ }^{15}$ Contamination of the preservation solution occurs frequently and is usually associated with the time of excision. ${ }^{4}$

The presence of contamination of the solution for organ preservation, at a rate of $27.9 \%$ as evidenced by this research, is comparable to data from other centers.

In all four preservation solutions used in this study, the presence of microbial growth was observed. The vast majority of the isolated agents observed were coagulase-negative Staphylococcus, reaching accounted for $29 \%$ of infections. When comparing this prevalence with that of other centers, it was observed that a large majority of isolated organisms 
are also coagulase-negative Staphylococcus. These microbes are of low virulence and rarely cause a significant infectious process. ${ }^{4,8}$ The high incidence of these agents allows us to propose that their origin may be from breach of aseptic techniques during collection. ${ }^{4}$

However, there is a possibility of contamination of the preservation solution with organisms of high virulence and multidrug resistance. Among these are Staphylococcus aureus, Pseudomonas spp., Klebsiella spp., Acinetobacter spp., Escherichia coli, Candida spp. and several others. ${ }^{4,5,17}$ All of the aforementioned agents were found in this study, totaling $71 \%$ of all microorganisms.

Cultures directed to anaerobic agents were not performed in this research. Many of the reported studies in the literature do not perform routine screening for anaerobes. The limited information available suggests low levels of infection by these agents in the recipient. ${ }^{16}$

The concomitant presence of infectious complications in the donor and positive culture of the organ preservation solution had a prevalence of $13.5 \%$ in this study. Univariate statistical analysis was not significant, but when the same variable was studied by the multivariate logistic regression model, it had statistical significance.

In a study conducted by Mattner et al., ${ }^{18}$ involving lung, heart-lung, heart and liver transplants, there were no significant differences between incidence of post-transplant infections in patients from the group receiving contaminated organs versus the group that received sterile organs.

Of the 89 deceased donors, 67 (75.3\%) of them have used antibiotics during the ICU stay. Many of these antibiotics were used empirically based on laboratory findings and non-specific clinical signs with no major relevance. Only 17 (19.1\%) donors who used antibiotics showed a growth in the culture of preservation solution. This finding showed statistical significance in its effectiveness to inhibit microbial growth by using antibiotics both in univariate and in multivariate logistic regression analyses. Unfortunately, in this study it was not possible to correlate the contaminant agent of the preservation solution with the infectious agent in the donor because donors' cultures were not routinely performed .

Of the 123 recipients enrolled in this study, 42 showed some infectious process, confirmed by culture performed up to the thirtieth day post-transplant.

Several studies have shown that the transmission of bacterial infections from the donor to the recipient is uncommon, ranging from 0 to $6.2 \% .{ }^{19}$ Mattner et al. ${ }^{18}$ showed that $3.9 \%(11 / 282)$ of the recipients developed postoperative infections with the same bacterial or fungal species that had grown in cultures of donor organs. Of these 11 species, six pairs were available for genotyping studies, and only one pair was proven to have the same genetic identity. Howev- er, these authors did not demonstrate significant statistical association between contamination of the donated organ, infection and mortality.

In a study conducted by Cerutti et al. ${ }^{5}$ mortality in the first year after transplant was significantly higher (5/11, $45 \%)$ when transmission of infection from donor to recipient occurred than when it did not (49/547, 9\%). In a study, Len et al. ${ }^{20}$ demonstrated that the transmission of infection from donor to recipient was documented in 5 of 292 patients (1.71\%), 2 of whom did not survive the infection (40\%). However, they found no difference in survival at 30 days among recipients who received organs from infected donors when compared to those who were not infected.

Coincidentally, it was noted that in two cases, the species of microorganism, isolated in cultures of the preservation medium was the same causing infections in the recipients. With respect to transmission of infection, objective evidence for the event can only be obtained by genotyping techniques, which would reveal the identity of microorganisms isolated in the donor and recipient. Unfortunately, such techniques are not widely available, and their application to microorganisms isolated in different laboratories is not feasible. ${ }^{5}$ In this study, the possible occurrence of transmission of the infectious agent from donor to recipient was only inferred when the same species was identified in both samples. Antimicrobial susceptibility could have also provided valuable information in this regard if it had been tested.

This study has limitations when attempting to relate causal factors for the presence of infection. Most of the data were collected in a previously established manner that, although performed according to a protocol, precludes collection of important data that could shed light on the incidence of contamination such as injury of the intestinal wall at the time of collection, duration of organ collection, contamination of surgical material or equipment, and antiseptic technique used. The literature is clear when it refers to collection as the major source of contamination. There is no information of possible complications during transport of the organ.

The collection of samples for culture immediately after harvesting could be important to compare with the sample at the time of transplant. These data could provide additional information on other possible time points of contamination.

In addition, quantitative analysis of the presence of microorganisms in the preservation solution, along with the conduction of antibiogram, could yield valuable information on whether or not contamination occurred during the collection process. So far, it is not clear what is the best way to determine the presence of contamination of the donated organ and when it occurred. ${ }^{18}$ In this paper the lack of statistical significance comparing recipients 
infection rates versus presence of contaminating microorganisms in the preservation solution may be explained by routine use of prophylactic antibiotics at the time of transplantation. Besides it should be taken into account the amount and virulence of contaminating microorganisms in the preservation solution.

Despite these limitations, this study provides important information for groups working with solid organ transplants. There was a prevalence of $27.9 \%$ of contamination by various agents in the liquid where the grafts were stored. Although most contamination was with agents of low virulence, microorganisms with high potential for morbidity of the organ recipient were found.

According to this paper and medical review on the issue, care must be taken in patients presenting signs of infection, especially those which preservation solution cultures from the transplanted organ yielded virulent microorganisms like Staphylococcus aureus, Pseudomonas spp., Klebsiella spp., Acinetobacter spp. and so on. In these cases the potential risk of a severe infection must be considered and attention is required to prescribe the most suitable antibiotics, especially in immune suppressed patients.

The non-use of antibiotics and the presence of infectious complications in the donor were significantly correlated with the presence of microorganisms in the preservation solution.

There is no connection between contamination of the solution for preserving organs and the presence of infectious complications in the recipient.

\section{REFERENCES}

1. Marinho A. Um estudo sobre as filas para transplantes no Sístema Único de Saúde brasileiro Cad Saúde Pública. 22[10], 2229-2239. 2006.

2. Bucher P, Mathe Z, Bosco D, et al. Microbial surveillance during human pancreatic islet isolation. Transplant Proc. 2004; 36:1147-1148.

3. Majeski JA, Alexander JW, First MR, Munda R, Fidler JP, Craycraft TK. Transplantation of microbially contaminated cadaver kidneys. Arch Surg. 1982; 117:221-224.
4. Wakelin SJ, Casey J, Robertson A, et al. The incidence and importance of bacterial contaminants of cadaveric renal perfusion fluid. Transpl Int. 2005; 17:680-686.

5. Cerutti E, Stratta C, Romagnoli R, Serra R. Bacterial- and fungalpositive cultures in organ donors: clinical impact in liver transplantation. Liver Transpl. 2006; 12:1253-1259.

6. Cox DR. Regression models and life-tables. J R Stat Soc Ser B. 1972;34(2):187-220. [10.0]. 2002. Chicago (IL), SPSS Inc.

7. Couto WJ, Carvalho AC, Branco JNR, et al. Transplante cardíaco e infecção. Bras Cir Cardiovasc. 2001; 16:141-151.

8. McCoy GC, Loening S, Braun WE, Magnusson MO, Banowsky LH, McHenry MC. The fate of cadaver renal allografts contaminated before transplantation. Transplantation. 1975; 20:467-472.

9. Lammermeier DE, Sweeney MS, Haupt HE, Radovancevic B, Duncan JM, Frazier OH. Use of potentially infected donor hearts for cardiac transplantation. Ann Thorac Surg. 1990; $50: 225$.

10. Wood RP, Melendez T, Langnas AN, Navakal H, Kelly D. The viability of microorganisms in preservation solutions. Transplantation. 1991; 51:239-242.

11. Snydman DR. Infection in solid organ transplantation. Transpl Infect Dis. 1999; 1:21-28

12. Rogers NM, Peh CA, Faull R, Pannell M, Cooper J, Russ GR. Transmission of toxoplasmosis in two renal allograft recipients receiving an organ from the same donor. Transpl Infect Dis. 2008; 10:71-74.

13. Linhares MM, Gonzalez AM, Triviño T, et al. Simultaneous pancreas-kidney transplantation: infectious complications and microbiological aspects. Transplant Proc. 2004; 36:980-981.

14. Little DM, Farrell JG, Cunningham PM, Hickey DP. Donor sepsis is not a contraindication to cadaveric organ donation. Q J Med. 1997; 90:641-642.

15. Haberal M, Demirag A, Cohen B, Turan M, Arslan G, Bilgin N. Microbiologic analysis of preservation solutions in cadaveric kidney transplantation. Transplant Proc. 1995; 27:2697-2698.

16. Gottesdiener KM. Transplanted infections: donor-to-host transmission with the allograft. Ann Intern Med. 1989; 110:1001-1016.

17. Delmonico FL, Snydman DR. Organ donor screening for infectious diseases: review of practice and implications for transplantation. Transplantation. 1998; 65:603-610.

18. Mattner F, Fischer S, Kola A, et al. Impact of bacterial and fungal donor organ contamination in lung, heart-lung, heart and liver transplantation. Infection. 2008; 36:207-212.

19. Zibari GB, Lipka J, Zizzi H, Abreo KD, Jacobbi L, McDonald JC. The use of contaminated donor organs in transplantation. Clin Transplantation. 2000; 14:397-400.

20. Len O, Gavalda J, Blanes M, et al. Donor infection and transmission to the recipient of a solid allograft. Am J Transplant. 2008; 8:2420-2425. 